\title{
INSTRUMENTO DE AVALIAÇÃO DO DESENVOLVIMENTO DE PRÉ-ESCOLARES DOS CENTROS DE EDUCAÇÃO E ALIMENTAÇÃO DO PRÉ-ESCOLAR *
}

\begin{abstract}
FERNANDES, J. \& GANDRA, Y. R. Instrumento de avaliação do desenvolvimento de pré-escolares dos Centros de Educação e Alimentação do Pré-Escolar. Rev. Saúde públ., S. Paulo, 15 (supl.):79-90, 1981.
\end{abstract}

RESUMO: Para testar os efeitos do programa dos Centros de Educação e Alimentação do Pré-Escolar (CEAPE) no desenvolvimento sócio-psico-pedagógico dos pré-escolares, foram estudados e selecionados itens de fácil obtenção e de sensibilidade suficiente para a estimativa da eficácia do programa nessa área. Estes itens constituiram o "Instrumento de Avaliação do Desenvolvimento do Pré-Escolar (IADPE)". Com o objetivo de se efetuar uma análise das principais características do "Instrumento", foi avaliado seu grau de discriminação e comparado seus resultados com os da "Escala de Comportamento" quando aplicados a crianças de 4,5 e 6 anos que frequentavam e as que não frequentavam o programa CEAPE. A análise dos resultados revelou que o "Instrumento" possui bom grau de discrinação; que seus itens variaram, sistematicamente, num mesmo sentido; e que quando considerados individualmente tenderam a correlacionar-se positivamente com o total do teste. O total do "Instrumento" apresentou correlações altamente significativas com o total da "Escala". O IADPE resultou num instrumento útil de avaliação psico-pedagógica de crianças de baixo nível sócio-econômico.

UNITERMOS: Pré-escolares, avaliação. Criança, desenvolvimento. Programa CEAPE.

\section{INTRODUÇÃO}

Para que o desenvolvimento integral dos pré-escolares ocorra de modo adequado são necessários, pelo menos, do:s fatores básicos: alimentação e estimulação ambiental satisfatórias. Estas duas condiçōes, entretanto, nem sempre podem ser totalmente satisfeitas pelo meio familiar em que o pré-escolar vive, não havendo, tampouco, uma assistência suficiente a ele por parte dos serviços públicos já existentes. Em consequiência, constata-se que os casos mais graves de desnutrição infantil ocorrem, geralmente, no primeiro ano de vida e na idade chamada pré-escolar e em crianças que são, na grande maioria dos casos, oriundas de famílias de baixo nível sócio-econômico. Analogamente, este mesmo quatro pode ser traçado quanto ao desen-

* Convênio 10/77 - INAN/DN/FSP/USP.

** Do Programa CEAPE - Departamento de Nutrição da Faculdade de Saúde Pública da USP.

*** Do Departamento de Nutrição da Faculdade ae Saúde Pública da USP - Av. Dr. Arnaldo, 715 - 01255 - São Paulo, SP - Brasil. 
FF'RNANDES, J. \& GANDRA, Y.R. Instrumento de avaliação do desenvolvimento de pré-escolares dos Centros de Educaçăo e Alimentação do Pré-Escolar. Rev. Saúde públ., S. Paulo, 15(supl.): 79-90, 1981.

volvimento de determinados aspectos psicológicos ${ }^{6}$, que são tidos como pré-requisitos essenciais para importantes eventos, como a escolarização, por exemplo. Nota-se, assim, que os anos pré-escolares fornecem os alicerces para outros acontecimentos importantes que ocorrerão nos anos seguintes. As deficiências nutricionais, aliadas ao desenvolvimento limitado de determinados aspectos psicológicos (exemplo: operações cognitivas e funções psico-neurológicas Poppovic e col. $\left.{ }^{14}, 1975\right)$, refletem-se nas altas taxảs de evasão e reprovação na primeira série do primeiro grau, que por sua vez geram outras conseqüências graves, ao nivel individual e social.

Em função destes pontos levantados $\mathrm{e}$ da sua gravidade em nosso país, são necessárias medidas urgentes factíveis, que permitam dar algum grau de contribuição para a solução desses problemas.

O programa CEAPE - Centros de Educação e Alimentação do Pré-Escolar (Gandra, 1973, 1976) 4,5, desenvolvido no interior do Estado de São Paulo, é um modelo alternativo para $o$ atendimento ao pré-escolar. Foi planejado para atender crianças entre 2 e 6 anos, fornecendo-lhes suplementação alimentar e atividades psico-pedagógicas, na forma de recreação orientada. O programa caracteriza-se pela praticidade, baixo custo e flexibilidade. Estes aspectos viabilizaram a expansão do programa, pois cada comunidade o implanta de acordo com os recursos de que dispõe, obedecendo, entretanto, um mínimo exigido e à orientaçōes básicas gerais do programa

Torna-se importante a tarefa de avaliação de programas pré-escolares alternativos mais simples como o CEAPE, pois eles são atualmente mais viáveis em termos de implantação a curto prazo e de atendimento a um grande número de crianças, conforme mostrou a expansão do modelo CEAPE, em várias áreas do país.

Para o CEAPE foram selecionados métodos não sofisticados de avaliação, porém adequados.
Em termos de avaliaçăo psico-pedagógica, procurou-se inicialmente um teste que discriminasse entre idades diferentes e que também pudesse ser utilizado para avaliar os efeitos educacionais do programa.

Por solicitação do Departamento de Nutrição da Faculdade de Saúde Pública da Universidade de São Paulo, o trabalho inicial foi desenvolvido por Poppovic e Campos ${ }^{12}, 1975$ que escolheram o teste "Preschool Inventory" (Caldwell ", 1970) como ponto de partida para a avaliação psico-pedagógica deste programa.

Foram realizadas, nos EUA, algumas pesquisas que utilizaram os testes de Caldwell 2 (bastante semelhantes ao "Preschool Inventory", em conteúdo) com o objetivo de avaliar a influência do programa Head Start sobre aspectos cognitivos e os resultados mostraram diferenças estatísticas significativas entre os grupos em estudo no teste de Caldwell, mas não no Quociente de Inteligência (QI). Estes resultados são compativeis com a filosofia que orientou a construção dos testes de Caldwell, que foi o de buscar um teste de aproveitamento sensivel, principalmente a aspectos relacionados com a escolarização, abordados num programa, como o Head Start, sem a preocupação de avaliar "inteligência", por exemplo.

Em princípio, então, este teste poderia ser escolhido como suficientemente sensivel para diferenciar duas populaçōes - uma formada por crianças atendidas por um programa de educaçăo pré-escolar e outra por crianças sem este tipo de atendimento.

As caracteristicas do "Preschool Inventory" foram consideradas bastante promissoras (Poppovic e Campos, 1975) 12, para o uso na avaliação de um programa como o CEAPE. Estes autores realizaram a adaptação inicial deste teste. Foram acrescentados itens de coordenação motora ampla (equilibrio), baseados na Escala de Desenvolvimento Motor de Ozeretzky, mantendo-se itens de avaliação de conceitos de cor, 
FERNANDES, J. \& GANDRA, Y.R. Instrumento de avaliação do desenvolvimento de pré-escolares dos Centros de Educação e Alimentação do Pré-Escolar. Rev. Saúde públ., S. Paulo, 15(supl.): 79-90. 1981

forma, tamanho, posição, número; itens de cópia, conhecimento de partes do corpo, do meio ambiente e execução de ordens. Faralelamente, também foi acrescentada uma versão resumida da "Escala de Comportamento Intra-Teste" (Poppovic e col., 1975) ${ }^{14}$, aqui chamada também de "Escala" utilizada para avaliar o comportamento da criança, em termos de sua relação com o examinador e com a tarefa, durante o teste. A adaptação de itens foi denominada "Inventário de Habilidade para Pré-Escolares" e foi testada, em outubro de 1975, com o objetivo básico de avaliar o grau de discriminação deste instrumento entre as idades de 4,5 e 6 anos. Os resultados obtidos encorajaram um trabalho mais intensivo com o "Inventário".

A versão inicial deste teste sofreu posteriormente uma nova adaptação, originando o "Instrumento de Avaliação do Desenvolvimento para Pré-Escolares - IADPE" ou, simplesmente neste trabalho, "Instrumento". Foram retirados itens pouco discriminados, isto é, os que apresentavam pequenos incrementos em termos de percentagens de acerto, ou pequenas variáveis ao longo das idades estudadas.

Paralelamente, foram acrescentados outros itens que permitissem uma amostragem mais abrangente dos conteúdos. Estes acréscimos foram realizados para aumentar o número de itens e a abrangência de certas áreas já existentes no teste e também para amostrar áreas ainda não representadas no instrumento (exemplo: analogias opostas, atenção e memória de curta duração, entre outros).

Em ambos os casos, os itens acrescentados foram baseados na literatura existente sobre a testagem de pré-escolares (Poppovic e col., 1975 14; Anastasi, $1967^{1}$; Kahn ${ }^{9,10}$; Szekely ${ }^{16}$, 1960), procurando-se manter a concepção original do instrumento.
Assim, o objetivo principal do presente trabalho é efetuar uma análise das características estatisticas principais do "IADPE", avaliando também o grau de discriminação apresentado por este instrumemnto e pela "Escala de Comportamento", entre as idades de 4,5 e 6 anos, quando aplicados a crianças "Ceapenses" e "Não-Ceapenses".

\section{MATERIAL E METODOS}

\section{Caracteristicas dos instrumentos}

O "IADPE" ou simplesmente "Instrumento", segue a mesma orientação que os testes originais de Caldwell ${ }^{2}$, ou seja, este instrumento é um teste de aproveitamento que pretende avaliar alguns aspectos elementares, considerados básicos para a escolarização*.

O "IADPE" contém 70 itens, sendo também aplicada a "Escala de Comportamento Intra-Teste"11.

A aplicação do material é individual e leva cerca de $25 \mathrm{~min}$ por criança. Os seus 70 itens cobrem os seguintes aspectos: conhecimento de partes do corpo, de cores; conceitos básicos (quantidade, tamanho, posição, entre outros); atenção e memória de curta duração, conceitos numéricos simples, reconhecimento de formas geométricas, equilibrio estático e dinámico. cópia de figuras geométricas e analogias opostas. Todos os itens admitem apenas as respostas "certo" (valor 1) ou "errado" (valor zero). Estes valores somados constituem o "Total do Instrumento", cujo valor máximo é 70 pontos.

A "Escala de Comportamento Intra-Teste" é utilizada para avaliar o comportamento da criança, com relação ao examinador e à tarefa, após a aplicação dos 70 itens iniciais. Esta "Escala" inclui 6 itens: quantidade de fala, persistência, atenção, auto-confiança, "rapport" e participação durante o teste. Cada item é avaliado pelo exami-

* o Manual de Instruções e o material do "Instrumento de Avaliação do Desenvolvimento do Pré-Escolar" podem ser encontrados no Departamento de Nutrição da Faculdade de Saúde Pública da Universidade de São Paulo. 
FE'RNANDES, J. \& GANDRA, Y.R. Instrumento de avaliação do desenvolvimento de pré-escolares dos Centros de Educação e Alimentação do Pró-Escolar. Rev. Saúde públ., S. Paulo, 15(supl.): $79-90,1981$.

nador numa escala de 1 a 5 pontos (discretos), definidos operacionalmente. $\mathrm{O}$ ponto 1 corresponde à pior realização possivel no item e o ponto 5 , à melhor realização. Estes valores são somados e constituem 0 "Total da Escala", cujo máximo é 30 pontos.

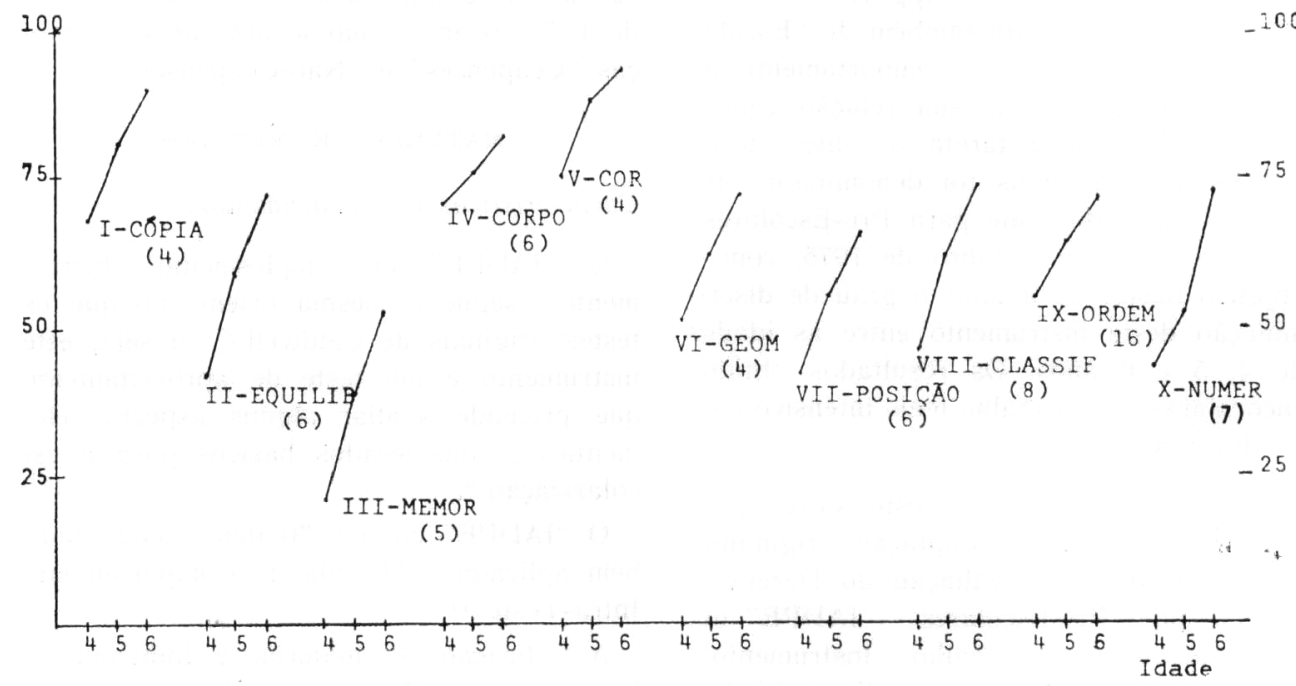

Fig. 1 - Resultados médios de cada agrupamento, no grupo "ceapense" expressos em percentagem do total máximo de pontos do agrupamento (entre parênteses, na figura).

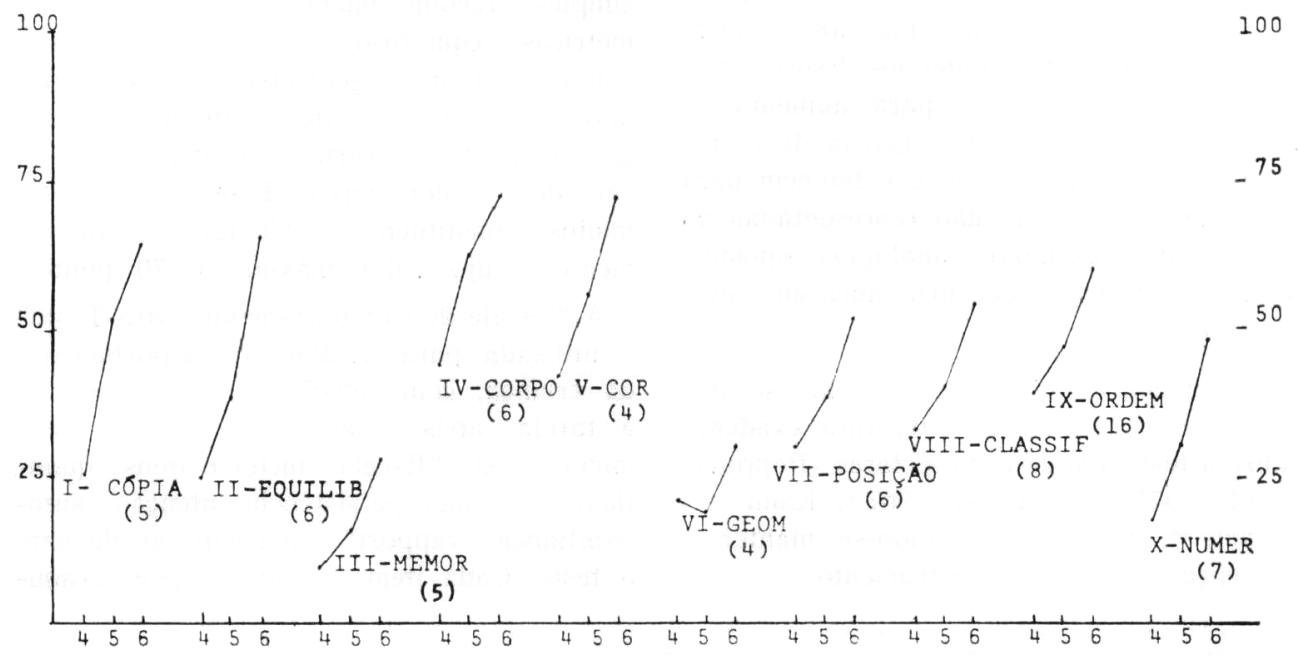

Fig. 2 - Resultados médios de cada agrupamento, no grupo "não-ceapense", expressos em percentagem do total máximo de ponfos do agrupamento (entre parênteses, na figura). 
FERNANDES, J. \& GANDRA, Y.R. Instrumento de avaliação do desenvolvimento de pré-escolares dos Centros de Educação e Alimentação do Pre-Escolar. Rev. Saúde públ., S. Paulo, 15(supl.): $79-90,1981$.

\section{Critérios de Seleção da Amostra}

Em vista da expansão do programa e da variabilidade existente, em termos de funcionamento entre os CEAPEs do Estado de São Paulo, optou-se por concentrar os trabalhos de avaliação psico-pedagógica na cidade de Leme. Esta localidade contava, no início dos trabalhos de avaliação, com cerca de 12 CEAPEs operando sob supervisão educacional direta da Prefeitura local.

O "IADPE" e a "Escala de Comportamento" foram aplicados a crianças de 4,5 e 6 anos. Para cada idade, foram formados dois grupos: um de crianças que frequientavam o programa CEAPE ("Ceapenses") e um grupo de crianças "Não-Ceapenses". As crianças Ceapenses foram selecionadas com base na idade cronológica e no tempo de frequiência ao programa (pelo menos 6 meses letivos seguidos, com freqüência de no mínimo $\mathbf{7 5 \%}$ ).

As crianças "Não-Ceapenses" não freqüentavam nenhum tipo de creche ou pré-escola e residiam em áreas próximas aos CEAPEs utilizados*, sendo originárias, no geral, de familias de baixo nível sócio-econômico.

\section{Descrição dos grupos}

Foram testadas 353 crianças "Ceapenses" e 214 "Não-Ceapenses"', com idades de 4, 5 e 6 anos. O número de crianças de cada grupo, por idade, está definido em trabalho anterior (Tabela 1) ${ }^{3}$.

Algumas informaçōes referentes ao nivel sócio-econômico familiar foram coletadas em entrevista com a mãe ou responsável pela criança, e já apresentadas em trabalho anterior ${ }^{3}$. Resumindo-se, pode-se dizer que ambos os grupos de crianças - "Ceapenses" e "Não-Ceapenses" - foram originária no geral, de famílias de baixo nível sócio-econômico.

\section{RESULTADOS}

Serāo considerados alguns aspectos analisados com relação ao grau de discriminação do "IADPE" e da "Escala de Comportamento", entre as idades de 4,5 e 6 anos.

Estas análises foram feitas separadamente para os grupos "Ceapense" e "Não-Ceapense”, dada a existência de diferenças entre elas, no que se refere a medidas de tendência central e de variabilidade ${ }^{3}$.

\section{Médias, Desvios-Padrões e Medianas do Total do "IADPE"}

De um modo geral, em ambos os grupos, as percentagens de acerto aumentaram, na grande maioria dos itens, com o aumento das idades $* *$. Obviamente, estes aumentos refletem-se no total dos resultados do "Instrumento".

As médias aritméticas, os desvios-padrão amostrais e medianas do total do "IADPE", para as diferentes idades dos grupos estudados podem ser verificados em trabalho anterior (Tabela 7$)^{3}$.

Pode-se notar que tanto os valores médios como os medianos aumentaram em função da idade. Análises iniciais mostraram que as variâncias dos diversos grupos foram significativamente diferentes, o que invalidaria o uso de procedimentos de Análise de Variância para a verificação dos efeitos da idade sobre o total do "Instrumento" (Keppel, 1973)11. Assim para a comparação das diferentes idades em cada grupo, optou-se pela prova não-paramétrica de KruskalWallis (Siegel, 1975) ${ }^{15}$. Foram feitas análises separadas para os grupos "Ceapense" e "Não-Ceapenses" e, em ambos os casos, encontravam-se valores altamente significativos $\left(\mathrm{H}_{\text {ceapenses }}=75,739 ; \mathrm{H}_{\text {não-ceapense }}\right.$ $\left.=66,993 ; \mathrm{X}_{\mathrm{c}}^{2}, 2 \mathrm{~g} .1=13,815, \mathrm{p}<.001\right)$. Rejeitou-se, portanto a hipótese de equivalência dos valores do total do "Ins-

* Detalhes sobre a seleção da amostra podem ser encontrados no artigo "Avaliação Inicial dos Efeitos Psico-Pedagógicos do CEAPE" 2.

* As tabelas das percentagens de acerto de cada item, por idade, podem ser encontradas na Comissão Central do CEAPE - Departamento de Nutrição da Faculdade de Saúde Pública da USP. 
FERNANDES, J. \& GANDRA, Y.R. Instrumento de avaliação do desenvolvimento de pré-escolares dos Centros de Educação e Alimentaçäo do Pré-Escolar, Rev, Saúde públ., S. Paulo, 15(supl.): $79-90,1981$.

trumento", nas diferentes idades, em cada grupo.

Análises subseqüentes, através da prova não -paramétrica de Mann-Whitney (Siegel, 1975) ${ }^{15}$, revelaram, em ambos os grupos, diferenças altamente significativas entre as idades de 4 e 5 anos, e entre 5 e 6 anos. A Tabela 1 mostra os valores de $Z$ obtidos através da prova de Mann-Whitney.
Assim, os resultados obtidos indicaram que o "IADPE" discriminou significativamente entre as idades de 4,5 e 6 anos, tanto para o grupo "Ceapense" como para o "Nāo-Ceapense".

Em ambos os grupos, pode-se observar uma tendência crescente praticamente linear, em função da idade, em termos de resultados medianos. Isto significa que o número de

T A B E L A 1

Valores de $\mathbf{Z}$ obtidos, através da prova de Mann-Whitney para as comparaçes do total do "Instrumento", entre as diferentes idades. (As probabilidades associadas aos valores de $Z$ são $p<.001$ ).

\begin{tabular}{l|cc}
\hline & Idades & \\
\hline Grupo & $4-5$ anos & $5-6$ anos \\
\hline Ceapennse & 3,89 & 6,27 \\
Não-Ceapense & 4,27 & 4,80 \\
\hline
\end{tabular}

pontos ganhos, em termos de valores medianos do "Total", foi bastante constante de uma idade para outra, dentro de um mesmo grupo.

Aparentemente, no grupo "Ceapense" existe uma tendência à concentração nos resultados próximos ao total máximo, com o aumento da idade (médias crescentes e diminuição dos desvios-padrões). Isto indica que o "IADPE" tende a ser mais fácil na idade de 6 anos, no grupo "Ceapense" $€$ que, em função deste aumento de "facilidade" do instrumento, ele diminui neste caso o seu grau de discriminação.

Agrupamentos dos Itens do "Instrumento de Avaliação do Desenvolvimento do Pré-Escolar - IADPE"

Com o objetivo de se estudar melhor o comportamento das áreas nos diferentes grupos etários, foram definidos 10 agrupamentos mutuamente exclusivos, com os itens do "IADPE". Este proceder apresentou algumas dificuldades, já que os vários itens avaliam mais de um aspecto. Assim, as partições envolveram critérios pessoais, em alguns casos. A orientação geral seguida foi reunir itens que avaliem predominantemente características em comum.

Os agrupamentos definidos, o total máximo possivel de pontos para cada agrupamento e as características avaliadas foram apresentadas em trabalho anterior (Tabela $8)^{3}$. As Figuras 1 e 2 apresentam as médias de pontos obtidas em cada agrupamento. expressas em percentagens do seu total máximo, para cada idade em cada grupo.

Todos os agrupamentos, com exceção do VI - Geometria (Não-Ceapenses" entre 4 e 5 anos) apresentaram médias de pontos crescentes, em função ao aumento da idade, tanto para o grupo "Ceapense" como para o "Não-Ceapense".

Estes resultados mostram, portanto, que as diferenças observadas entre as idades, no total do "Instrumento" não são atribuíveis apenas à alguns agrupamentos específicos, mas refletem diferenças de desempenho, entre as idades, ao longo de todo 0 teste. Estas diferenças entre as idades foram observadas tanto no grupo "Ceapense" como no "Não-Ceapense". Embora possuam valores diferentes para cada grupo, elas indicam que o "IADPE", considerado no seu 
FERNANDES, J. \& GANDRA, Y.R. Instrumento de avaliação do desenvolvimento de pré-escolares dos Centros de Educação e Alimentação do Pré-Escolar. Rev. Saúde públ., S. Paulo, 15(supl.): $79-90,1981$.

"Total" ou em agrupamentos, foi sensivel aos efeitos da idade.

\section{Médias das correlaçōes Item-Total ("IADPE")}

Os valores da correlação entre um determinado item e o total do teste fornecem uma avaliação da "validade" do item (Guilford, 1954) ${ }^{8}$, ou seja, indicam qual o grau em que um determinado item varia no mesmo sentido que os demais, tomados em conjunto. Assim, as correlaçōes item-total são essencialmente medidas de homogeneidade (Anastasi, 1976) ${ }^{1}$. De um modo geral, considera-se que a média das correlações item-total fornece uma medida do poder de discriminação do teste entre crianças de alto e baixo desempenho (Poppovic e Campos, 1975) ${ }^{13}$.

A Tabela 2 apresenta as médias aritméticas das estimativas dos valores de correlação ponto-bisserial * entre a nota de cada item (zero ou 1) e o total do "Instrumento", excluída a nota do referido item. Estas estimativas foram obtidas empregando-se a fórmula de correção proposta por Guilford $(1954)^{8}$ nos valores originais das correlações. A correção foi utilizada para eliminar aumentos indevidos nos valores das correlações, decorrentes do fato de se correla- cionar a nota de um item com um total, do qual a nota deste item faz parte. As médias ** destes valores foram calculadas através da transformação $Z$ de Fisher (Guilford, 1956) ${ }^{i}$ e são apresentadas na Tabela 5 para cada grupo e idade, separadamente.

Esta Tabela mostra que as médias das estimativas das correlações item-total foram positivas e significativas, em todas as idades. Isto indica que os itens do "IADPE" variaram, no geral, sistematicamente no mesmo sentido que o conjunto dos demais, discriminando satisfatoriamente entre as crianças que obtiveram altos e baixos resultados no final do teste, dentro de cada idade.

\section{Coeficientes de Precisão $(K R-20)$ do "IADPE"}

A Tabela 3 mostra os valores dos coeficientes de precisão do "IADPE", segundo a fórmula KR-20 (Guilford, 1954) ${ }^{8}$, para os diferentes grupos de idade. Num sentido amplo, a precisão de um teste indica o grau em que as diferenças individuais nos resultádos do teste são atribuiveis a erros casuais de mensuração e o grau em que elas são atribuíveis a diferenças verdadeiras, existentes entre os individuos, nas características em consideração (Anastasi, 1967) ${ }^{1}$. O coeficiente de precisão KR-20 é um dos vários

T A B E L A 2

Médias das correlações item-total, por grupo e idade. (Todas as médias de correlaçõeg apresentaram $(\mathrm{p}<.001)$, com exceção do grupo Ceapense de 4 anos $(\mathrm{p}<.01)$,

\begin{tabular}{l|ccc}
\hline & & & \\
Grupo & 4 anos & 5 anos & 6 anos \\
\hline Ceapense & & & 0,231 \\
Não-Ceapense & 0,357 & 0,261 & 0,338 \\
\hline
\end{tabular}

* Para obtenção dos valores das correlações item-total, no caso de testes formados por itens dicotômicos, os cálculos feitos a partir das fórmulas de correlação produto-momento de Pearson fornecem os mesmos resultados numéricos que os obtidos através das fórmulas de correlação ponto-bisserial (Tavella, 1978. pág. 68)1i.

** Os valores da correlação ponto-bisserial, para cada item em cada grupo e idade podem ser encontrados na Comissão Central dos CEAPEs. (Departamento de Nutrição da Faculdade de Saúde Pública da Universidade de São Paulo). 
FIRNANDES, J. \& GANDRA, Y.R. Instrumento ds: avaliação do desenrolvimento de pré-escoiares dos Centros de Educação e Alimentação do Pré-Escolar, Rev. Saúde públ., S. Paulo, 15(supl.): $79-90,1981$.

métodos utilizados para a avaliação da consistência interna de um teste. Este coeficiente indica o grau de inter-correlação entre os itens e é, portanto, também uma medida de homogeneidade do teste.

Conforme mostra a Tabela 3 , os coeficientes de precisão variaram entre 0,85 e 0,92 . Estes resultados permitem classificar a precisão (consistência interna) do "Instrumento" entre "boa" e "ótima", de acordo com os padrões tradicionalmente aceitos.

\section{Erros Padróes de Medida}

O erro padrão de medida (Guilford, $1954)^{\delta}$ está intimamente associado com o coeficiente de precisão. A utilização dos erros-padrão parte do pressuposto de que a nota total do "Instrumento", obtida por uma determinada criança, envolve algum grau de erro de mensuração.

A Tabela 4 mostra os valores dos erros-padrão de medida para cada grupo e idade estudada.
Supondo-se, por exemplo, uma criança "Ceapense" de 5 anos que obtenha 45 pontos no total do "Instrumento", existe uma faixa entre $45-3,5$ pontos e $45+3,5$ pontos que, com uma probabilidade de cerca de 0,68 , contém o "Total" real desta criança.

Conforme mostra a Tabela 4 , os errospadrão de medida variaram entre 3,0 e 3,5 pontos para as diferentes idades dos grupos, o que indica que o grau de erro envolvido, ao longo das idades, é razoavelmente estável.

Médias, Desvios-Padrões e Medianas do total da "Escala"

Conforme já foi levantado, a "Escala de Comportamento Intra-Teste" 14 é utilizada para avaliar o comportamento da criança com relação ao aplicador e à tarefa, durante o teste. Ela é formada por 6 itens, cada um recebendo uma ponderação de 1 a 5 pontos. Portanto, o total máximo da "Escala" é 30 pontos.

T A B E L A 3

Coeticientes de precisão (fórmula KR-20) do "Instrumento" aplicado aos grupos "Ceapense" e "Não-Ceapense".

\begin{tabular}{lccc}
\hline \multicolumn{1}{c}{ Grupo } & \multicolumn{2}{c}{ Idade } \\
& 4 anos & $\mathbf{5}$ anos & 6 anos \\
\hline Ceapense & 0.92 & 0,85 & 0.88 \\
Não-Ceapense & 0.89 & 0,91 & 0.91 \\
\hline
\end{tabular}

$T A B E I A$

Erros padrōes de medida do "Instrumento" aplicado aos grupos "Ceapense" e "Não-Ceapense".

\begin{tabular}{lccc}
\hline Grupo & 4 anos & $\begin{array}{c}\text { Idade } \\
5 \text { anos }\end{array}$ & 6 anos \\
\hline Ceapense & 3,3 & 3.5 & 3.0 \\
Năo-Ceapense & 3,2 & 3,4 & 3.4 \\
\hline
\end{tabular}


FERNANDES, J. \& GANDRA, Y.R. Instrumento dc avaliação do desenvolvimento de pré-escolares dos Centros de Educação e Alimentação do Pré-Escolar, Rev. Saúde públ., S. Paulo, 15(supl.): $79-90,1981$.

As médias, desvios-padrões e medianas do "Total da Escala de Comportamento", para as diferentes idades dos grupos estudados, podem ser verificados em trabalho anterior, na Tabela $9^{3}$.

Novamente pode-se observar que os resultados médios e medianos obtidos aumentaram em função da idade, em ambos os grupos ("Ceapense" e "Não-Ceapense"). Por outro lado, existiu também uma diminuição da variabilidade em função do aumento da idade, o que sugere que os resultados concentraram-se cada vez mais nos valores mais altos da "Escala" ("efeito de limite").

As diferenças entre idades foram novamente testadas através da prova não-paramétrica de Kruskal-Wallis (Siegel, 1975) ${ }^{15}$, separadamente para o grupo "Ceapense" e "Não-Ceapense". Os resultados altamente significativos, em ambos os grupos, mostraram que as três idades não receberam avaliações equivalentes na "Escala" $\mathrm{H}$ ceapenses $=30,032, \mathrm{H}$ não-ceapense $=24,752, \mathrm{X}^{2}{ }_{\mathrm{c}}$, $2 \mathrm{~g} .1=13,815, \mathrm{p}<.001$ ), e que, portanto, existiram diferenças significativas entre pelo menos duas das três idades pesquisadas.

Foram feitas análises posteriores, através da prova não-paramétrica de Mann-Whitney. Estas análises revelaram, em ambos os grupos, diferenças significativas entre as idades de 4 e 5 anos, e entre 5 e 6 anos.
A Tabela 5 mostra os valores de $Z$ obtidos através desta prova.

Dessa forma, os resultados obtidos indicaram que o total da "Escala de Comportamento" discriminou significativamente entre as idades de 4,5 e 6 anos, em ambos os grupos, embora houvesse uma tendência a concentração de resultados nos valores mais próximos ao valor máximo do instrumento, com o aumento da idade.

\section{Correlações "IADPE" - "Escala"}

A Tabela 6 mostra os valores da correlação não-paramétrica de Spearman (Siegel, 1975)15 entre o total do "Instrumento" e o total da "Escala", para as diferentes idades de cada grupo.

\section{T A B E L A 5}

Valores de $\mathbf{Z}$ obtidos*, através da prova de Mann-Whitney, para as comparações do "total da escala" entre as diferentes idades de grupos "Ceapense" e "Não-Ceapense".

\begin{tabular}{lcc}
\hline \multicolumn{1}{c}{ Grupo } & \multicolumn{3}{c}{ Idades } \\
& $4-5$ anos & $5-6$ anos \\
\hline Ceapense & 3,32 & 2,46 \\
Não-ceapense & 2,19 & 3,40 \\
\hline Todos os valores são significativos ao nível \\
de .05.
\end{tabular}

T A B E L A 6

Valores da correlação de Spearman * entre o "total do instrumento" e o "total da escaia de comportamento" dos grupos "Ceapense" e "Não-Ceapense".

\begin{tabular}{lccc}
\hline Grupo & 4 anos & $\begin{array}{c}\text { Idade } \\
\text { (5 anos }\end{array}$ & 6 anos \\
\hline Ceapense & 0,508 & 0,438 & 0,376 \\
Não-Ceapense & 0,608 & 0,524 & 0,416 \\
\hline
\end{tabular}

- Todos os valores são significativos, com $\mathrm{p}<.001$ 
FERNANDES, J. \& GANDRA, Y.R. Instrumento de avaliação do desenvolvimento de pré-escolares dos Centros de Educação e Alimentação do Pré-Escolar. Rev. Saúde públ., S. Paulo, 15(supl.): $79-90,1981$.

Todos os valores foram altamente significativos $(\mathrm{p}<.001)$ e tenderam a diminuir com o aumento da idade, em ambos os grupos. Aparentemente, estes decréscimos nos valores das correlações foram decorrentes do "efeito de limite" observado na "Escala de Comportamento". Ou seja, com o aumento da idade, os resultados do total da "Escala" como era de se esperar concentravam-se cada vez mais nos valores próximos ao seu máximo. Não houve, portanto, no grupo de 6 anos variabilidade suficiente para que o total da "Escala" pudesse manter correlações com o total do "IADPE" tão altas quanto as verificadas aos 4 anos.

\section{DISCUSSÃO E CONCLUSÃO}

Conforme já foi levantado, as análises foram feitas separadamente para o grupo "Ceapense" e "Não-Ceapense" da cidade de Leme (SP), uma vez que houve diferenças nitidas entre eles quanto a medidas de tendência central e de variabilidade. Este procedimento permitiu, portanto, a avaliação mais sistemática das diferentes características do instrumento, nas diferentes idades.

A análise de alguns aspectos estatísticos do "IADPE" revelou que, nos grupos estudados, este instrumento possui bom grau de discriminação entre e dentro das idades pesquisadas. $O$ teste apresentou coeficientes de precisão (consistência interna) bastante satisfatórios. Pode-se afirmar, então, que, de um modo geral, seus itens variaram sistematicamente num mesmo sentido, com boas inter-correlações entre si. Como conseqüência, os itens, considerados isoladamente, tenderam a se correlacionar positivamente com o total do teste.
No caso do grupo Ceapense, aos 6 anos, o teste tendeu diminuir seu grau de discriminação, na medida em que aumenta a sua "facilidade". Isto sugere que, nesta idade, o teste tende a um "efeito de limite" (concentração nos valores próximos ao máximo possível).

Em ambos os grupos, os resultados da "Escala de Comportamento" também apresentaram diferenças significativas entre as idades estudadas. Aos 5 anos, o total da "Escala" já começou a concentrar-se nos valores próximos ao seu máximo ("efeito de limite"); mesmo assim foram encontradas diferenças significativas entre 5 e 6 anos, em ambos os grupos.

As correlações altamente significativas entre o total do "Instrumento" e o total da "Escala" são compativeis com resultados existentes na literatura (Anastasi, 1967) ${ }^{1}$ acerca das relações entre o comportamento e atitudes do examinando, na situação de avaliação e o seu resultado final no teste. Estes resultados reproduziram também os obtidos por Poppovic e Campos, $1975^{12}$ e Poppovic e col.14, 1975, em que foram encontradas correlaçōes significativas entre a "Escala" e testes psico-pedagógicos.

As análises feitas em termos de aspectos estatísticos, juntamente com a seleção e adaptação prévias dos conteúdos dos itens, apontam para a adequação do "IADPE" como instrumento útil de avaliação psico-pedagógica de pré-escolares, de 4 a 6 anos, de baixo nivel sócio-econômico. É óbvio, entretanto, que ele deve ser utilizado e interpretado dentro dos limites fixados por seus objetivos, pelo conteúdo dos seus itens e pelas próprias características decorrentes de uma testagem breve. 
FERNANDES, J. \& GANDRA, Y.R. Instrumento de avaliação do desenvolvimento de pré-escolares dos Centros de Educação e Alimentação do Pré-Escolar. Rev. Saúde públ., S. Paulo, 15(supl.): $79-90,1981$.

FERNANDES, J. \& GANDRA, Y. R. [An instrument for the assessment of the development of preschool children (IADPE) in the CEAPE Program]. Rev. Saúde vúbl.. S. Paulo, 1s(suppl.):79-90, 1981.

ABSTRACT: Selected items, with adequate sensibility to evaluate the eficacy of the CEAPE program with regard to the psycho-pedagogical development of preschool children were tested. These selected items together composed the "Instrumento de Avaliação do Desenvolvimento do Pré-Escolar - IADPE". The objective of this paper was to study statiscally the principal characteristics of that "Instrument" evaluating its capacity of discrimination and comparing its results to those obtained on a "Behavior Scale" for preschoolers. The results show that the "Instrument" correlated well with those of the "scale" ana that it is a very useful and practical system for the evaluation of the psycho-pedagogical development of preschoolers.

UNITERMS: Preschool child, evaluation. Child development. CEAPE Program.

\section{REFERENCIAS BJBLIOGRAFICAS}

1. ANASTASI. A. Testes psicológicos. São Paulo, Ed. Herder, 1967.

2. CALDWELL, B.M. Cooperative preschool inventory. Ed. rev. Princeton, N.J., Educational Testing Service, 1970.

3. FERNANDES, J. \& GANDRA, Y.R. Avaliação inicial dos efeitos psico-pedagógicos dos Centros de Educação e Alimentação do Pré-Escolar. Rev. Saúde públ., S. Paulo, 15(supl.) : 64-78, 1981.

4. GANDRA, Y.R. Asistencia alimentaria por medio de centros de educación y alimentación del pre-escolar. Bol. Ofic. sanit. panamer., 74:302-14, 1973.

5. GANDRA, Y.R. Nutrition and dietetic education program for preschool children at primary schools in Brazil. In: Anderson, M.A. \& Grewal, T., ed. Nutrition planning in developing world: Proceedings of Regional Workshops held by CARE in India, Kenya and Colombia, 1976. Bogotá, Programas Editoriales, 1976. p. 205-12.
6. GANDRA, Y.R. O pré-escolar de dois a seis anos de idade e o seu atendimento. Rev. Saúde públ., S. Paulo, 15 (supl.): 3-8, 1981.

7. GUILFORD, J.P. Fundamental statistics in psychology and education. New York. McGraw-Hill, 1956.

8. GUILFORD, J.P. Psychometric methods. New York, McGraw-Hill, 1954.

9. KAHN, T.C. Kahan intelligence tests: experimental form. Perceptual and Motor Skill, (Monogr. suppl.):10-1; 123-53, 1960.

10. KAHN, T.C. A new culture - free test. Psychol. Rep., 6:239-42, 1960.

11. KEPPEL, G. Design and analysis: a researcher's handbook. Englewood Clifts, N.J., Prentice-Hall, 1973.

12. POPPOVIC, A.M. \& CAMPOS, M.M.M. Inventario de habilidades para pré-escolares; relatorio. Sảo Paulo, Departamento de Pesquisas Educacionais. Fundação Carlos Chagas. 1975. 
FERNANDES, J. \& GANDRA, Y.R. Instrumento de avaliação do desenvolvimento de pré-escolares dos Centros de Educação e Alimentação do Pré-Escolar. Rev. Saúde públ., S. Paulo, 15(supl.): $79-90,1981$.

13. POPPOVIC, A.M. \& CAMPOS, M.M.M. Inventário de habilidades para pré-escolares; relatorio do Pré-Teste. São Paulo, Departamento de Pesquisas Educacionais. Fundação Carlos Chagas, 1975.

14. POPPOVIC, A.M. et al. Marginalização cultural: subsidios para um curriculo pré-escolar. Cad. Pesq. Fund. Carlos Chagas, 14:7-74, 1975.

15. SrEGEL, S. Estatística nāo-paramétrica para as ciências do comportamento. São Paulo, McGraw-Hill do Brasil, 1975.
16. SZEKELY, B. Los testes. Buenos Aires, Ed. Kapelusz, 1960.

17. TAVELLA, N.M. Analises de los itens en la construción de instrumentos psicométricos. México, Ed. Trillas, 1978.

Recebido para publicąão em 10/07/1981

Aprovado para publicação em 1\%/11/1981 